\title{
PCSK9 and Atherosclerosis - Lipids and Beyond
}

\author{
Michael D. Shapiro and Sergio Fazio
}

Oregon Health \& Science University, Knight Cardiovascular Institute, Center for Preventive Cardiology, Portland OR, USA

Even though it is only a little over a decade from the discovery of proprotein convertase subtilisin/kexin type 9 (PCSK9) as a plasma protein that associates with both high and low cholesterol syndromes, a rich body of knowledge has developed, and drugs inhibiting this target have been approved in many markets. While the majority of research in recent years has focused on the impact of therapeutic antagonism of this molecule, important lines of investigation have emerged characterizing its unique physiology as it relates to cholesterol metabolism and atherosclerosis. The PCSK9 story is unfolding rapidly but is far from complete. One chapter that is of particular interest is the possible direct link between PCSK9 and atherosclerosis. This review specifically examines this relationship drawing from data produced from experimental models of plaque biology and inflammation, atherosclerosis imaging studies, and observational epidemiology.

Key words: Proprotein convertase subtilisin/kexin type 9, Low density lipoprotein, Hypercholesterolemia, Lipid metabolism, Atherosclerosis, Inflammation

\section{Introduction}

The history of the initial discovery and characterization of proprotein convertase subtilisin/kexin type 9 (PCSK9) has been well described ${ }^{1)}$. The seminal discovery in 2003 by Abifadel et al. linked mutations in the gene encoding PCSK9 with autosomal dominant hypercholesterolemia $(\mathrm{ADH})^{2}$. This finding uncovered a key new player in cholesterol homeostasis and set into motion intensive research in the field of PCSK9 biology. The basic construct places this extracellular secretory protein as a central regulator of plasma low-density lipoprotein (LDL)-cholesterol (LDL-C) concentration. PCSK9, by direct interaction with the hepatic LDL receptor (LDLR), enhances its degradation by targeting it for destruction in the lysosome, disallowing its natural recycling loop ${ }^{3)}$. The fundamental observation that PCSK9 is intrinsically linked to LDLR recycling and familial hypercholesterolemia $(\mathrm{FH})$ provided the basis for pursuing it as a therapeutic target. PCSK9 causes degradation of the LDLR, thus inhibiting PCSK9 prolongs the lifespan of the LDLR, which leads to drastic

Address for correspondence: Sergio Fazio, Oregon Health and Science University, 3181 SW Sam Jackson Park Rd, Portland, OR 97239, USA

E-mail: fazio@ohsu.edu

Received: February 4, 2017

Accepted for publication: February 26, 2017 reductions in plasma LDL-C levels. Major findings over the last decade have revealed the following:

(a) Gain-of-function mutations in PCSK9 are a cause of $\mathrm{ADH}^{2)}$.

(b) Loss-of-function mutations in PCSK9 are associated with low LDL-C levels and markedly reduced cardiovascular risk ${ }^{4-77}$.

(c) Therapeutic antagonism of PCSK9 reduces LDL-C levels and, within the context of clinical trials, appears safe, efficacious, and well tolerated ${ }^{8)}$.

Beyond the obvious association of PCSK9induced LDLR degradation and hypercholesterolemia, emerging studies have uncovered other mechanisms by which PCSK9 may facilitate atherosclerosis development independent of its impact on lipids. What follows is a survey of the literature that provides insight into the impact of PCSK9 on atherosclerosis via lipid and non-lipid pathways.

\section{PCSK9 and Lipoprotein Metabolism}

The classic teachings on lipoprotein metabolism place regulation of cholesterol squarely within the cell, with all the components available to sense and react to cholesterol depletion and excess. The discovery and elucidation of PCSK9 has revolutionized our understanding of lipid metabolism and has introduced this secreted circulatory protein as a central actor in this process. Comprehensive reviews have described mech- 
anisms by which PCSK9 impacts the LDLR and ultimately plasma LDL-C ${ }^{8-10)}$. Complete knockout of PCSK9 in mice results in severe hypocholesterolemia with reductions in LDL cholesterol of up to $80 \%$ and reductions in total cholesterol of up to $40 \%{ }^{4,11,12)}$. Despite the profound hypocholesterolemia, these mice are viable and fertile. Interestingly, mice that are engineered with liver specific PCSK9 knock down demonstrate a complete absence of plasma PCSK9 corroborating the notion that the liver, despite being only one of several tissues that synthesizes PCSK9, is the primary source of its plasma levels ${ }^{13)}$. This observation suggests that PCSK9 produced from extrahepatic tissues may have effects that are either exclusively intracellular, or extracellular but only paracrine, or both. However, even in the complete absence of plasma PCSK9, the liver specific PCSK9 knock down model only demonstrates an approximately $30 \%$ reduction in total plasma cholesterol levels, a significantly attenuated phenotype compared to complete knockout of PCSK9. This finding suggests that PCSK9 secreted from the liver exerts a dominant, but not complete, effect on plasma cholesterol levels, and provides impetus to study the role of extrahepatic PCSK9 in wholebody cholesterol homeostasis ${ }^{4,12)}$.

Murine models incorporating a human PCSK9 transgene under an ApoE promoter overexpress PCSK9 and are found to be healthy, with normal reproductive capacity, and display the anticipated severe hypercholesterolemia phenotype ${ }^{12,14)}$. As compared to LDLR knockout $(\mathrm{KO})$ mice, which have a 15-fold increase in plasma LDL-C, this PCSK9 overexpression model exhibits a more modest (5-fold) increase in plasma LDL-C despite the fact that, as in the LDLR KO model, there is no demonstrable LDLR in the liver ${ }^{12)}$. This observation again highlights the importance of extrahepatic tissues in cholesterol metabolism and suggests the following:

1. Extrahepatic LDLR plays a central role in LDL-C catabolism

2. Extrahepatic LDLR is not influenced by plasma PCSK9

As new data emerge, it has become evident that the impact of PCSK9 on lipids and atherosclerosis is transduced by mechanisms beyond its effect on plasma LDL clearance. PCSK9 also targets receptors beyond the LDLR, including the VLDLR, CD36, ApoER2, and LRP ${ }^{15-17)}$. The extent to which its action on these LDLR family members is significant for cholesterol homeostasis remains unclear, though there are several relevant observations relating to triglyceride-rich lipoprotein (TRL) metabolism. Given the importance of TRL in the initiation and propagation of atherosclerosis, the impact of PCSK9 on these highly atherogenic particles may provide another mechanism by which therapeutic antagonism of PCSK9 may reduce the risk of atherosclerotic cardiovascular disease (ASCVD).

Plasma PCSK9 concentration correlates to fasting triglyceride levels in males and females, though not in the obese ${ }^{18-23)}$. Individuals with the PCSK9 S127R gain-of-function mutation demonstrate increased levels of all apoB100-containing lipoproteins, including VLDL and remnants ${ }^{24)}$. Furthermore, therapeutic antibodies to PCSK9 reduce plasma triglycerides, though this effect appears to be more modest relative to the LDL-C reduction and as compared to the statin effect on TG vs. LDL-C ${ }^{25}$, 26). Since apoB turnover is heavily modulated by LDLR-mediated reuptake of many apoB-containing lipoproteins, therapeutic PCSK9 inhibition also impacts plasma TRL concentrations. A large proportion of newly synthesized apoB is degraded within the secretory pathway by diversion to the autophagic compartment ${ }^{27)}$. Thus, the rate of apoB secretion, and hence, VLDL secretion, from the liver is determined by the proportion of apoB that escapes post-translational degradation ${ }^{28)}$. In addition, reuptake of newly secreted lipoproteins also regulates the net output of $\mathrm{apoB}^{29)}$. Twisk et al. demonstrated that the LDL receptor influences the posttranslational fate of apoB by increasing presecretory apoB degradation and mediating reuptake of nascent lipoprotein particles ${ }^{30)}$. In this context, PCSK9-mediated degradation of hepatic LDLR can cause hypertriglyceridemia by liberating apoB from LDLR-induced intracellular catabolism as well as reducing uptake of nascent apoB-containing lipoproteins (eg, VLDL). Indeed, several experimental models have demonstrated that PCSK9 directly increases hepatic production of apoB-containing lipoproteins. Acute adenoviral-mediated overexpression of PCSK9 in fasting mice leads to profoundly increased hepatic production of apoB and VLDL-triglycerides ${ }^{31)}$. Similarly, chronic hepatic expression of PCSK9 leads to overproduction of VLDL in transgenic mice. Murine expression of human PCSK9 led to increases in hepatic lipogenesis in both an apoE and LDLR-dependent manner ${ }^{32)}$. Moreover, changes in hepatic PCSK9 production correspond to changes in VLDL production ${ }^{33)}$. Lastly, PCSK9 deficient mice demonstrate decreased postprandial triglyceride levels ${ }^{34)}$. Shunting of TRL from plasma to visceral adipose tissue in the absence of PCSK9 may be due to its effects on VLDLR and CD36, which are upregulated in the perigonadal fat depots of female PCSK9-knockout mice. Absence of PCSK9 leads to preservation of these two surface proteins which in turn facilitates fatty acid uptake into adipose tissue and causes adipocyte hypertrophy ${ }^{13)}$. Despite these empiric observations, the mechanisms 
that govern PCSK9 regulation on TRL secretion are not fully understood. Interestingly, a recent study in mice lacking LDLR demonstrated that PCSK9 did not affect the expression of genes and proteins involved in hepatic lipogenesis (both SREBP- and non-SREBP-mediated), findings that differ from what was seen in the intestine ${ }^{32,35,36)}$.

The intestine is only second to the liver in production of PCSK9 and similarly has an impact on intestinally derived plasma TRL, which are produced during meal absorption and contain apoB48 as main structural protein. Mechanistic studies demonstrated that PCSK9 increases the production and secretion of intestinal TRL by both transcriptional and post-transcriptional mechanisms involving both the sterol-regulatory element-binding proteins (SREBP) and nonSREBP pathways, with subsequent lipid accumulation in enterocytes ${ }^{34-37)}$. In murine knockout models, the absence of PCSK9 was associated with a reduction in apoB48 secretion and attenuation of postprandial hypertriglyceridemia ${ }^{11,34)}$. Given the significant atherogenic impact of chylomicron remnants, this observation may represent additional therapeutic potential of PCSK9 inhibitors to reduce the risk of ASCVD. Beyond intestinal TRL metabolism, PCSK9 reduces cholesterol absorption by decreasing expression of NPC1L1, the main entry point of cholesterol into the enterocytes from the intestinal lumen ${ }^{36)}$. Furthermore, recent results suggest that PCSK9 increases the intestinal, not the hepatic, contribution to plasma cholesterol and triglyceride levels in the absence of LDLR ${ }^{32}$. Lipoprotein(a) $[\mathrm{Lp}(\mathrm{a})]$ is a low density lipoprotein (LDL) particle consisting of a molecule of apolipoprotein(a) (a variant protein with a highly heterogeneous number of plasminogen kringle 4 repeats) covalently bound to apolipoprotein $\mathrm{B}^{38}$. Epidemiological and genetic data have consistently shown $\mathrm{Lp}(\mathrm{a})$ to be an independent risk factor for ASCVD ${ }^{39-43)}$. Therapeutic monoclonal antibodies to PCSK9 have been found to reduce $\mathrm{Lp}(\mathrm{a})$ by about $25-30 \%$, irrespective of baseline levels by a yet unidentified mechanism ${ }^{44,45)}$. This observation is compelling, as $\operatorname{Lp}(\mathrm{a})$ is a highly atherogenic particle with no specific therapy to lower its plasma levels and thus has generated renewed interest in elucidating the molecular and cellular link between PCSK9, LDLR, and Lp(a) metabolism. While many have postulated that the observed $\mathrm{Lp}(\mathrm{a})$ lowering is due to extreme upregulation and clearance via the LDLR, this is difficult to reconcile in light of seminal work demonstrating that the LDLR is not a physiologically important route of $\mathrm{Lp}(\mathrm{a})$ catabolism in humans ${ }^{46}$. Furthermore, a recent study demonstrated that the effect of PCSK9 on $\mathrm{Lp}(\mathrm{a})$ is related to reduced secretion rates, rather than its cellular uptake ${ }^{47)}$. We previously demonstrated that plasma PCSK9 preferentially associates with $\operatorname{Lp}(\mathrm{a})$ particles in humans with elevated $\mathrm{Lp}(\mathrm{a})^{48)}$. In this context, injection of antiPCSK9 mAbs may lead to the formation of immune complexes on the surface of $\operatorname{Lp}(\mathrm{a})$ particles that may be cleared by mechanisms unrelated to normal $\mathrm{Lp}(\mathrm{a})$ clearance routes ${ }^{10)}$.

\section{PCSK9 and Inflammation}

PCSK9 induced perturbations of lipid metabolism and ultimately plasma lipid levels are the most obvious mechanism that links PCSK9 to atherosclerosis. A recent line of investigation has sought, however, to determine if PCSK9 itself has a primary role in atherosclerotic plaque development, independent of lipid changes. Results of such studies, summarized below, have made it clear that the proatherosclerotic action of PCSK9 is not entirely due to its impact on plasma concentrations of apoB containing lipoproteins, at least in the mouse model.

Several years ago, investigators demonstrated that PCSK9 is expressed in endothelial cells, vascular smooth muscles cells (VSMC), and in other areas of the human atherosclerotic plaque ${ }^{49)}$. Furthermore, VSMC expression of PCSK9 leads to cleavage of LDLR at the surface of arterial macrophages ${ }^{49)}$. Additional study revealed that VSMC produce considerably more PCSK9 than do endothelial cells, and that expression is dependent on shear stress, with maximal secretion at the lowest levels of shear stress corresponding to the known correlation between shear and arterial branch points (eg, bifurcations are vulnerable areas) ${ }^{50)}$. In a Pcsk9 $9^{-1-}$ murine model of vascular injury, placement of a non-occlusive collar on the carotid artery was associated with reduced neointimal formation and atherosclerotic lesions with reduced VSMC and collagen, though without a difference in macrophage content ${ }^{51)}$.

An interesting facet of the association between inflammation, PCSK9, and atherosclerosis relates to LOX-1, an important receptor for oxidized-LDL (oxLDL). LOX-1 expression is upregulated in the inflammatory state. In $\mathrm{LDLR}^{-/}$mice fed a high cholesterol diet, atherosclerosis and arterial wall inflammation are reduced with deletion of $L O X-1^{52,53)}$. Interestingly, the crosstalk between PCSK9 expression and LOX-1 in VSMC leads to a feed-forward loop where PCSK9 stimulates transcription of $L O X-1$ and LOX-1 activation stimulates PCSK9 expression ${ }^{54)}$. The interaction between PCSK9 and LOX-1 may facilitate atherogenesis, particularly during inflammatory states. Whether PCSK9 inhibitors can inhibit atherogenesis by antagonizing LOX-1 expression remains to be seen. 
In murine models of overexpression of either normal or gain-of-function PCSK9, increased atherosclerotic plaque size was observed and explained by the increase in plasma levels of atherogenic lipoproteins ${ }^{55,56)}$. As might be anticipated, the extent of expression of PCSK9 in $\mathrm{LDLR}^{-1-}$ mice has little to no effect on plasma lipids and atherosclerosis ${ }^{57,58)}$. Interestingly, while the absence of PCSK9 in apoE $\mathrm{E}^{-/-}$mice has a negligible impact on plasma lipids and atherosclerosis, the overexpression of PCSK9 has negligible effects on plasma lipids but is associated with increased atherosclerotic lesion size ${ }^{56,57)}$. In a series of experiments, Tavori et al. investigated the mechanisms of action of human PCSK9 on hepatic lipid and lipoprotein production on atherosclerosis using a previously developed line of transgenic mice expressing human PCSK9 within the normal physiologic range in human plasma (30-3000 ng/mL) ${ }^{19,59-62)}$. Specifically, plasma lipids, hepatic lipogenesis, and atherosclerotic lesion size and composition were evaluated in these transgenic mice expressing human (h) PCSK9 (hPCSK9) on a wild-type (WT), $\mathrm{LDLR}^{-/-}$, or apoE ${ }^{-/-}$background. Plasma apoB, cholesterol, and triglyceride levels increased in the WT mice as a result of decreased hepatic LDLR and increased hepatic lipid production. These changes were mediated both transcriptionally and post-transcriptionally by PCSK9 in both an LDLR- and apoE-dependent manner. Although in mice expressing hPCSK9 but lacking either LDLR or apoE no significant changes in plasma lipids were seen, hPCSK9 expression increased aortic lesion size in the absence of apoE but not in the absence of LDLR. Furthermore, the atheromatous plaque in apoE $\mathrm{E}^{-/-}$mice was enriched in hPCSK9 and Ly6C hi (inflammatory) monocytes. These findings establish that PCSK9 significantly affects atherosclerotic lesion size and by mechanisms that are not exclusively related to systemic lipid changes. PCSK9 promotes atherosclerosis directly by modulation of the entry of inflammatory monocytes into the artery wall by PCSK9LDLR interaction in the plaque. As an extension, this supports the notion that therapeutic antagonism to PCSK9 may improve ASCVD outcomes through both lipid and non-lipid pathways.

Further validation of the role of PCSK9-induced plaque inflammation, independent of plasma lipid changes, comes from studies of apoE ${ }^{-/-}$and $\mathrm{LDLR}^{-/-}$ mice expressing hPCSK9 from bone marrow-derived cells $^{63)}$. Such a model allows investigation of the direct effect of PCSK9 in the atheroma without confounding by plasma lipoproteins. While these mice manifested similar lipid levels and atherosclerotic lesion size, plaque composition demonstrated an LDLRdependent increase in $\mathrm{Ly}_{6} \mathrm{C}^{\mathrm{hi}}$ monocytes. Interest- ingly, these compositional changes are observed in the background of a very small macrophage contribution to plasma PCSK9 (0.5-1.5\% of total plasma PCSK9), supporting the notion that the plaque may be enriched by macrophage-derived PCSK9 rather than being bathed in plasma PCSK9. Additionally, expression of both pro- and anti-inflammatory cytokines was significantly modulated by macrophage hPCSK9 in an LDLR-dependent fashion.

It is important to consider the findings of these studies in a broader context. Specifically, previous work has demonstrated that pro-inflammatory leukocytes modulate atherosclerotic lesion composition and are an important factor in progression of atherosclerosis, despite similar plasma cholesterol levels and lesion size $^{64)}$. Moreover, lesion composition rather than size favors plaque rupture and thrombosis ${ }^{65)}$. Therefore, based on new experimental observations, one can hypothesize that, even in the absence of changes in lipid levels, hPCSK9 directly contributes to atherogenesis by altering plaque morphology and increasing inflammatory Ly6C $\mathrm{C}^{\text {hi }}$ monocyte infiltration and differentiation in the plaque. It is tempting to speculate that the absence of LDLR should mitigate the negative effect of PCSK9 on inflammation since PCSK9 accumulation in tissues depends on its presence ${ }^{60)}$. However, other LDLR family members, such as LRP1, play a role in inflammation and atherogenesis and interact with PCSK9 (Fig. 1). PCSK9 leads to degradation of LRP1 in macrophages which may, in part, explain the relationship of PCSK9 and atherosclerosis, as macrophage LRP1 deficiency is associated with increased atherosclerosis and plaque inflammation $^{63,66)}$.

\section{PCSK9 and Atherosclerosis Imaging}

The experimental studies reviewed above provide mechanistic insight into the relationship of PCSK9 and atherosclerosis. As a first step in translation, a series of atherosclerosis imaging studies have been performed exploring the association between serum PCSK9 and atherosclerotic plaque burden. Chan et al performed carotid intima-media wall thickness (CIMT) measurements in 295 asymptomatic subjects from the community ${ }^{67)}$. They found a significant association between PCSK9 and CIMT that was independent of traditional cardiovascular risk factors, including gender, hypertension, smoking, LDL-C, triglycerides, Lp(a), obesity, and biomarkers of inflammation. These findings are in agreement with smaller studies investigating the association of PCSK9 and CIMT ${ }^{68,69)}$. On the other hand, a sub-analysis of the FATE study (Firefighters and Their Endothelium) evaluated 1,527 


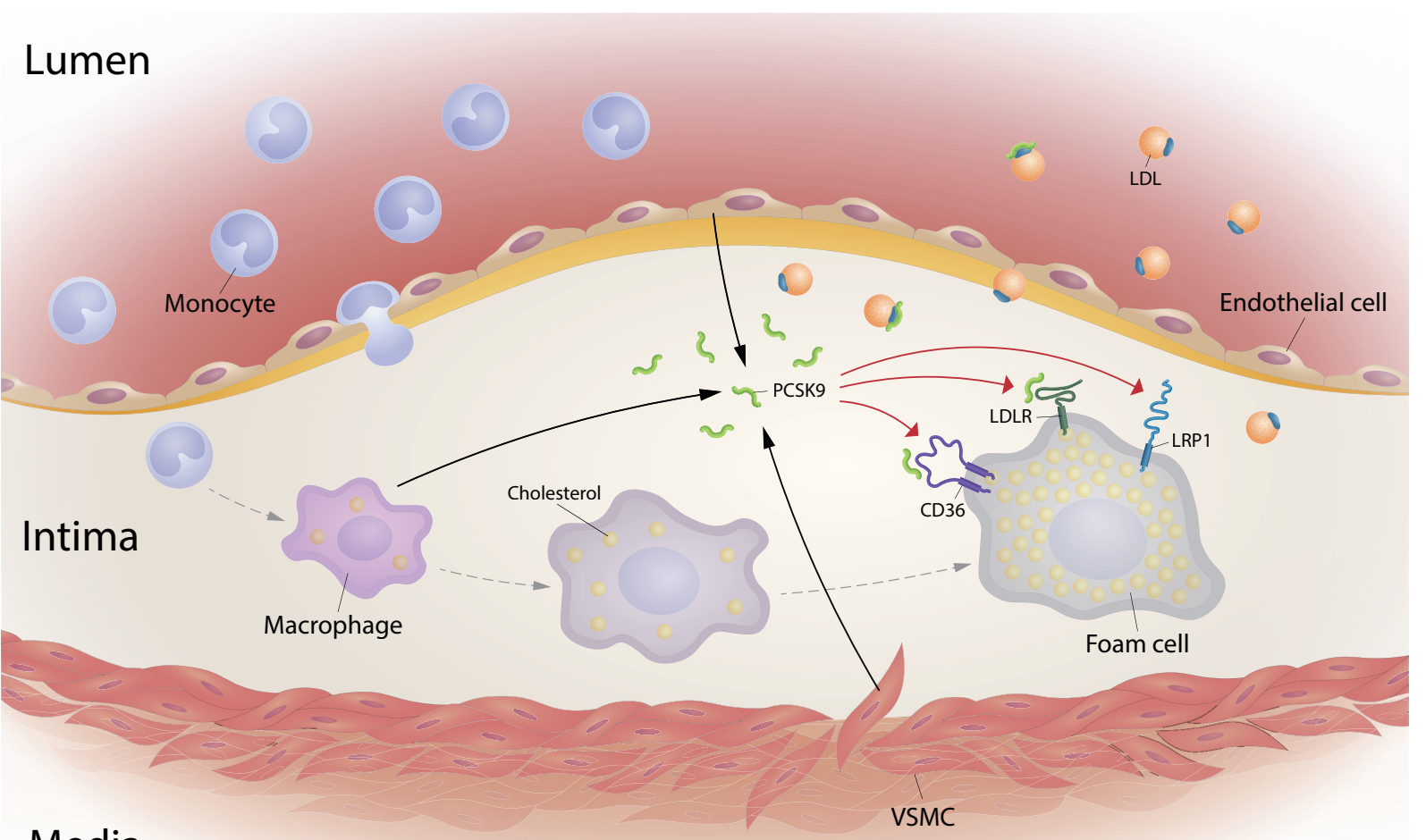

\section{Media}

Fig. 1. Sources and targets of intraplaque PCSK9

PCSK9 can be produced locally within atherosclerotic plaque by endothelial cells, vascular smooth muscle cells, and arterial macrophages. Intraplaque PCSK9 exerts paracrine effects on an array of foam cell receptors, including the LDL receptor, LRP1, and CD36. While local production of PCSK9 within the plaque has the potential to modulate atherogenesis independent of plasma concentrations, its impact on each of these foam cell receptors in relationship to atherosclerosis remains unclear.

PCSK9 = proprotein convertase subtilisin kexin type 9; LDL =low-density lipoprotein; LDLR =low-density lipoprotein receptor; LRP1 = Low density lipoprotein receptor-related protein 1; CD36=cluster of differentiation 36; VSMC=vascular smooth muscle cell

middle-aged men free of vascular disease who underwent risk factor assessment, including advanced biomarkers, flow-mediated dilation, and $\mathrm{CIMT}^{70)}$. While PCSK9 concentration was associated with several traditional risk factors, there was no relationship between PCSK9 levels and measures of subclinical atherosclerosis.

Computed tomography for quantification of coronary artery calcium (CAC) has also been used as a tool to evaluate the association of PCSK9 and atherosclerosis. Alonso et al. studied a cohort of 161 genetically confirmed subjects with FH who had plasma measurements of PCSK9 and underwent CAC scoring ${ }^{71)}$. They found that serum PCSK9 concentrations were independently predictive of CAC. Specifically, serum PCSK9 concentrations were significantly lower and higher in subjects without and with the highest absolute CAC scores, respectively. Perhaps most interesting, after correction for potential confounders, including age, gender, body mass index, fasting glucose, statin therapy, tobacco use, hypertension, and lipid/lipoprotein parameters, only PCSK9 and apo(a) remained significantly predictive of CAC scores in this cohort of asymptomatic FH subjects.

Cheng et al. utilized a novel approach to see if they could recapitulate experimental findings regarding PCSK9-induced inflammation and atherosclerosis by employing intravascular ultrasound virtual histology (IVUS-VH) as a tool in humans ${ }^{72}$. They evaluated the association of serum PCSK9 levels with necrotic core within coronary atherosclerotic lesions in subjects with known CAD confirmed at the time of angiography. They found that PCSK9 concentrations were linearly associated with a higher necrotic core fraction in coronary plaque. This observation remained significant in all subgroups, independent of LDL-C levels and use of statins.

There is limited literature to reflect on with regards to serial imaging studies. Xie et al. evaluated the association of serum PCSK9 with progression of carotid plaque as part of the Chinese Multi-provincial Cohort Study ${ }^{73)}$. The investigators found that among 
643 participants from the general population free of cardiovascular disease at baseline, PCSK9 levels were associated with progression of atherosclerosis as reflected by total plaque area, independent of plasma LDL-C concentrations. More recently, the GLAGOV (GLobal Assessment of Plaque reGression With a PCSK9 antibOdy as Measured by intraVascular Ultrasound) trial evaluated the effects of the PCSK9 inhibitor, evolocumab, on progression of coronary atherosclerosis in statin treated patients using seral IVUS ${ }^{74)}$. GLAGOV is a multicenter, double-blinded, placebocontrolled trial, which randomized subjects with angiographic coronary artery disease on baseline statin therapy to receive monthly evolocumab $(n=484)$ or placebo $(n=484)$. IVUS was performed at baseline and then at 76 weeks to assess changes in coronary atherosclerosis. They found a greater decrease in percent atheroma volume (1\% difference) in the group that received evolocumab. Additionally, treatment with evolocumab was associated with plaque regression in a greater percentage of patients than placebo (64.3\%vs 47.3\%; difference, $17.0 \% ; P<.001$ ). This study demonstrates that PCSK9 inhibition with aggressive LDL-C lowering is associated with a modest degree of plaque regression. Whether this finding is related to the aggressive LDL-C lowering (achieved mean, time-weighted LDL-C on evolocumab/statin was $36.6 \mathrm{mg} / \mathrm{dl}$ ), other non-lipid anti-atherosclerotic effects of PCSK9, or both, remains to be elucidated.

\section{PCSK9 as a Biomarker of ASCVD Risk}

Given the results from experimental and imaging studies that demonstrate an important relationship between PCSK9 and atherogenic lipids, inflammation, and atherosclerosis, there has been a natural interest in exploring PCSK9 as a biomarker of ASCVD risk, both in the context of primary and secondary prevention. As part of the FATE study, 1,527 middle aged firefighters who were free of vascular disease at baseline were followed longitudinally for a mean of $7.2 \pm 1.7$ years to evaluate the ability of PCSK9 concentrations to predict cardiovascular events $^{70)}$. While PCSK9 correlated with LDL-C, insulin, and triglycerides, it did not demonstrate a significant relationship with cardiovascular events. Ridker $e t$ al. performed a nested case-control evaluation from a prospective cohort of 28,000 initially healthy American women, $\geq 45$ years old and not on statin therapy, who participated in the Women's Health Study ${ }^{75)}$. The investigators measured plasma concentrations of PCSK9 at baseline among 358 cases (MI, ischemic stroke, CV death) and 358 age, smoking, and hormone replacement therapy matched controls (remained free of disease during 17 years of followup). The median PCSK9 concentrations were not statistically different amongst cases vs control (304.4 s 299.7), respectively. As expected, there was a modest, positive association between PCSK9 level and apoB and triglycerides. While baseline apoB predicted the occurrence of first cardiovascular events, baseline levels of PCSK9 did not. More recently, Leander et al. enrolled 4,232 sixty-year old men and women living in Stockholm County in a prospective cohort study examining the relationship between PCSK9 and future cardiovascular events (composite primary outcome of fatal or non-fatal myocardial infarction, angina, chronic ischemic heart disease, sudden cardiac death, and fatal or non-fatal ischemic stroke) ${ }^{76}$. During the 15 years of follow-up, the cumulative incidence of the primary outcome was $13 \%$. Not surprisingly, there was a modest correlation between PCSK9 levels with LDL cholesterol $(r=0.18, p<0.0001)$ and triglycerides $(r=0.12, p<0.0001)$. They found improved cardiovascular disease event-free survival in subjects in the lowest quartile of PCSK9 and the highest hazard ratios for incident cardiovascular disease in those in the highest quartile of PCSK9. One particularly interesting sub-analysis demonstrated that subjects with discordant PCSK9 and LDL-C levels (high PCSK9 - low LDL-C) had the highest future hazard of the primary outcome, even compared to those with high PCSK9 - high LDL-C. Additionally, the authors demonstrated improved discrimination (modestly improved c-statistic) and net reclassification with the incorporation of PCSK9 levels into their model (though neither was statistically significant). How do we reconcile the findings in this study with prior work? It is important to note significant differences in the study populations and methods. In particular, Leander et al. used a customized ELISA assay that found a much lower median PCSK9 levels than the Ridker cohort $(94.3 \mathrm{ng} / \mathrm{mL}$ vs $-300 \mathrm{ng} / \mathrm{mL}$, respectively). It must be noted that Leander et al. enrolled a representative sample of men and women from the general Swedish population whereas Ridker et al. studied a sub-cohort from a randomized controlled trial in women who were assigned to treatment with aspirin or vitamin $\mathrm{E}$ for prevention of cardiovascular disease.

In the context of secondary prevention, Werner et al. tested whether fasting PCSK9 serum concentrations predict events in a large cohort of statin-treated patients with well-controlled LDL-C levels and angiographically documented stable coronary artery disease ${ }^{77)}$. The study team enrolled 504 subjects with clinically stable coronary artery disease. These subjects were followed prospectively for the primary composite outcome including cardiovascular death, acute coro- 
nary syndrome (ACS), or unplanned, symptominduced coronary revascularization. Interestingly, while serum PCSK9 levels predicted the primary outcome in subjects on statin therapy and well controlled LDL-C levels, the association was lost when adjusting for fasting triglyceride levels. Perhaps this observation is not surprising when taking into account the influence of PCSK9 on other non-LDL atherogenic lipoprotein fractions. Li et al. studied 616 Chinese subjects with stable coronary artery disease for 17 months to evaluate the association of PCSK9 levels with the composite primary outcome of cardiac death, stroke, myocardial infarction, revascularization, and unstable angina $^{78)}$. These subjects were not on lipid-lowering therapy prior to enrollment but received medical therapy without or with percutaneous coronary intervention after enrollment. Interestingly, they found an association between PCSK9 levels and coronary artery disease severity. Specifically, there were significantly higher PCSK9 levels observed in subjects with a SYNTAX score in the highest vs the lowest tertile. Furthermore, at 17 months the event rate was higher in those with higher PCSK9 levels, but evident only among subjects treated medically, and not those who underwent coronary revascularization. The prognostic value of PCSK9 levels has also been studied in patients with ACS. Gencer et al. measured PCSK9 concentrations in 2030 subjects admitted with ACS undergoing coronary angiography ${ }^{79)}$. The participants were followed up at 30 days and 1 year after the event for the primary endpoint of all-cause death. They observed that PCSK9 concentrations in subjects with ACS were associated with measures of inflammation, lipid-lowering therapy, and the clinical onset of ACS. However, high PCSK9 levels did not associate with 1-year mortality.

Clearly, on the basis of the various trials evaluating PCSK9 as a biomarker of ASCVD risk, there are discrepant results from which no firm conclusions can be drawn. On that basis, a group of investigators performed a systematic review and meta-analysis of studies evaluating the relationship of PCSK9 levels with the risk of future cardiovascular events ${ }^{80)}$. Ultimately, 8 studies were included in their analysis, all published very recently, and with 12,081 subjects followed for a mean of 6.6 years. The main finding from this analysis is that PCSK9 levels are modestly but significantly correlated with risk of total cardiovascular events. Specifically, high vs. low PCSK9 levels are associated with a $23 \%$ higher risk for total cardiovascular events. Furthermore, an increase in PCSK9 levels by 1 SD corresponds to a $10 \%$ increase in total cardiovascular events. Based on the available data, acquisition of plasma PCSK9 levels cannot be endorsed at this time for clinical use. Its association with ASCVD events is modest and less robust than other commonly used and more validated biomarkers. However, these analyses highlight the need for standardization and refinements in the methodologies to assay PCSK9. Currently, there are two commonly used methods to measure plasma PCSK9, ELISA (common and scalable) and mass spectrometry (expensive and cumbersome). Importantly, recent lines of experimental evidence have demonstrated that PCSK9 is compartmentalized within the plasma, with approximately $40 \%$ of PCSK9 bound to LDL or Lp(a) particles and the remainder circulating freely, or at least not associated with lipoproteins ${ }^{48,60,81,82)}$. Given the stoichiometry between LDL and PCSK9 in plasma, approximately only 1 LDL particle in every 500-1000 carries at least one molecule of PCSK9. LDL-bound PCSK9 appears to be the more biologically active from, with free plasma PCSK9 susceptible to furin cleavage, a form that is less biologically active. Currently available ELISA techniques are not able to discriminate the various forms of plasma PCSK9. Development of a practical and reproducible assay that can quantitate the different compartments of plasma PCSK9 may finally herald the birth of PCSK9 as a valuable clinical biomarker.

\section{Conclusion}

Examination of experimental, imaging, and epidemiological studies demonstrates that PCSK9 is inextricably linked to atherosclerosis through both lipid and non-lipid pathways. One important culmination of this work will perhaps be reflected by the results of the clinical trials testing the therapeutic monoclonal antibodies to PCSK9, the first of which is expected to be published in $2017^{83)}$. Preliminary analyses suggest that randomized controlled trials are indeed likely to demonstrate that antagonism of PCSK9 is associated with a significant reduction in ASCVD events ${ }^{74,84,85)}$. These trials, of course, cannot answer the question as to which and to what relative extent each of these mechanisms of PCSK9 inhibition thwarts atherosclerosis. Furthermore, the current clinical approach utilizing antibodies only inhibits plasma (hepatic, secreted, extracellular) PCSK9. Novel agents in development have the ability to inhibit production (eg, antisense oligonucleotides, silencing RNA) of PCSK9 and therefore may highlight the relative importance of intracellular PCSK9 on inflammation and atherosclerosis. We are still at the dawn of the PCSK9 era, and the future is bright! 


\section{Acknowledgements}

Drs. Shapiro and Fazio were partially supported by NIH R01HL132985. The authors gratefully acknowledge Ms. Deanna Plubell for assistance with the figure.

\section{Disclosures}

Dr. Fazio has received compensation for advisory activities from the following companies: Aegerion, Amarin, Amgen, Kowa, Merck, Sanofi.

Dr. Shapiro has received compensation for advisory activities from the following companies: Alexion, Amgen, Bracco, GE Health Care, Synta Pharmaceuticals.

\section{References}

1) Abifadel M, Elbitar S, El Khoury P, Ghaleb Y, Chemaly M, Moussalli ML, Rabes JP, Varret M and Boileau C. Living the PCSK9 adventure: from the identification of a new gene in familial hypercholesterolemia towards a potential new class of anticholesterol drugs. Curr Atheroscler Rep. 2014; 16: 439

2) Abifadel M, Varret M, Rabes JP, Allard D, Ouguerram K, Devillers M, Cruaud C, Benjannet S, Wickham L, Erlich D, Derre A, Villeger L, Farnier M, Beucler I, Bruckert E, Chambaz J, Chanu B, Lecerf JM, Luc G, Moulin P, Weissenbach J, Prat A, Krempf M, Junien C, Seidah NG and Boileau C. Mutations in PCSK9 cause autosomal dominant hypercholesterolemia. Nat Genet. 2003; 34: 154156

3) Zhang DW, Lagace TA, Garuti R, Zhao Z, McDonald M, Horton JD, Cohen JC and Hobbs HH. Binding of proprotein convertase subtilisin/kexin type 9 to epidermal growth factor-like repeat A of low density lipoprotein receptor decreases receptor recycling and increases degradation. J Biol Chem. 2007; 282: 18602-18612

4) Cohen J, Pertsemlidis A, Kotowski IK, Graham R, Garcia $\mathrm{CK}$ and Hobbs HH. Low LDL cholesterol in individuals of African descent resulting from frequent nonsense mutations in PCSK9. Nat Genet. 2005; 37: 161-165

5) Cohen JC, Boerwinkle E, Mosley TH, Jr. and Hobbs $\mathrm{HH}$. Sequence variations in PCSK9, low LDL, and protection against coronary heart disease. $\mathrm{N}$ Engl J Med. 2006; 354: 1264-1272

6) Hooper AJ, Marais AD, Tanyanyiwa DM and Burnett JR. The C679X mutation in PCSK9 is present and lowers blood cholesterol in a Southern African population. Atherosclerosis. 2007; 193: 445-448

7) Fasano T, Cefalu AB, Di Leo E, Noto D, Pollaccia D, Bocchi L, Valenti V, Bonardi R, Guardamagna O, Averna $\mathrm{M}$ and Tarugi P. A novel loss of function mutation of PCSK9 gene in white subjects with low-plasma low-density lipoprotein cholesterol. Arterioscler Thromb Vasc Biol. 2007; 27: 677-681

8) Dadu RT and Ballantyne CM. Lipid lowering with
PCSK9 inhibitors. Nat Rev Cardiol. 2014; 11: 563-575

9) Shapiro MD, Fazio $S$ and Tavori H. Targeting PCSK9 for therapeutic gains. Curr Atheroscler Rep. 2015; 17: 499

10) Shapiro MD and Fazio S. From Lipids to Inflammation: New Approaches to Reducing Atherosclerotic Risk Circulation Research. 2016; 118: 732-749

11) Rashid S, Curtis DE, Garuti R, Anderson NN, Bashmakov Y, Ho YK, Hammer RE, Moon YA and Horton JD. Decreased plasma cholesterol and hypersensitivity to statins in mice lacking Pcsk9. Proc Natl Acad Sci U S A. 2005; 102: 5374-5379

12) Zaid A, Roubtsova A, Essalmani R, Marcinkiewicz J, Chamberland A, Hamelin J, Tremblay M, Jacques H, Jin W, Davignon J, Seidah NG and Prat A. Proprotein convertase subtilisin/kexin type 9 (PCSK9): hepatocyte-specific low-density lipoprotein receptor degradation and critical role in mouse liver regeneration. Hepatology. 2008; 48: 646-654

13) Roubtsova A, Munkonda MN, Awan Z, Marcinkiewicz J, Chamberland A, Lazure C, Cianflone K, Seidah NG and Prat A. Circulating proprotein convertase subtilisin/kexin 9 (PCSK9) regulates VLDLR protein and triglyceride accumulation in visceral adipose tissue. Arterioscler Thromb Vasc Biol. 2011; 31: 785-791

14) Park SW, Moon YA and Horton JD. Post-transcriptional regulation of low density lipoprotein receptor protein by proprotein convertase subtilisin/kexin type 9a in mouse liver. J Biol Chem. 2004; 279: 50630-50638

15) Seidah NG. New developments in proprotein convertase subtilisin-kexin 9's biology and clinical implications. Curr Opin Lipidol. 2016; 27: 274-281

16) Poirier S, Mayer G, Benjannet S, Bergeron E, Marcinkiewicz J, Nassoury N, Mayer H, Nimpf J, Prat A and Seidah NG. The proprotein convertase PCSK9 induces the degradation of low density lipoprotein receptor (LDLR) and its closest family members VLDLR and ApoER2. J Biol Chem. 2008; 283: 2363-2372

17) Shan L, Pang L, Zhang R, Murgolo NJ, Lan H and Hedrick JA. PCSK9 binds to multiple receptors and can be functionally inhibited by an EGF-A peptide. Biochem Biophys Res Commun. 2008; 375: 69-73

18) Cariou B, Langhi C, Le Bras M, Bortolotti M, Le KA, Theytaz F, Le May C, Guyomarc'h-Delasalle B, Zair Y, Kreis R, Boesch C, Krempf M, Tappy L and Costet P. Plasma PCSK9 concentrations during an oral fat load and after short term high-fat, high-fat high-protein and highfructose diets. Nutr Metab (Lond). 2013; 10: 4

19) Lakoski SG, Lagace TA, Cohen JC, Horton JD and Hobbs HH. Genetic and metabolic determinants of plasma PCSK9 levels. J Clin Endocrinol Metab. 2009; 94: 2537-2543

20) Baass A, Dubuc G, Tremblay M, Delvin EE, O'Loughlin J, Levy E, Davignon J and Lambert M. Plasma PCSK9 is associated with age, sex, and multiple metabolic markers in a population-based sample of children and adolescents. Clin Chem. 2009; 55: 1637-1645

21) Janis MT, Tarasov K, Ta HX, Suoniemi M, Ekroos K, Hurme R, Lehtimaki T, Paiva H, Kleber ME, Marz W, Prat A, Seidah NG and Laaksonen R. Beyond LDL-C lowering: distinct molecular sphingolipids are good indicators of proprotein convertase subtilisin/kexin type 9 
(PCSK9) deficiency. Atherosclerosis. 2013; 228: 380-385

22) Kwakernaak AJ, Lambert G and Dullaart RP. Plasma proprotein convertase subtilisin-kexin type 9 is predominantly related to intermediate density lipoproteins. Clin Biochem. 2014; 47: 679-682

23) Sullivan S, Fabbrini E, Horton JD, Korenblat K, Patterson BW and Klein S. Lack of a relationship between plasma PCSK9 concentrations and hepatic lipoprotein kinetics in obese people. Transl Res. 2011; 158: 302-306

24) Ouguerram K, Chetiveaux M, Zair Y, Costet P, Abifadel M, Varret M, Boileau C, Magot T and Krempf M. Apolipoprotein B100 metabolism in autosomal-dominant hypercholesterolemia related to mutations in PCSK9. Arterioscler Thromb Vasc Biol. 2004; 24: 1448-1453

25) Koren MJ, Lundqvist P, Bolognese M, Neutel JM, Monsalvo ML, Yang J, Kim JB, Scott R, Wasserman SM, Bays $\mathrm{H}$ and Investigators M-. Anti-PCSK9 monotherapy for hypercholesterolemia: the MENDEL-2 randomized, controlled phase III clinical trial of evolocumab. J Am Coll Cardiol. 2014; 63: 2531-2540

26) Blom DJ, Hala T, Bolognese M, Lillestol MJ, Toth PD, Burgess L, Ceska R, Roth E, Koren MJ, Ballantyne CM, Monsalvo ML, Tsirtsonis K, Kim JB, Scott R, Wasserman SM, Stein EA and Investigators D. A 52-week placebocontrolled trial of evolocumab in hyperlipidemia. N Engl J Med. 2014; 370: 1809-1819

27) Borchardt RA and Davis RA. Intrahepatic assembly of very low density lipoproteins. Rate of transport out of the endoplasmic reticulum determines rate of secretion. J Biol Chem. 1987; 262: 16394-16402

28) Yao Z, Tran K and McLeod RS. Intracellular degradation of newly synthesized apolipoprotein B. J Lipid Res. 1997; 38: 1937-1953

29) Williams KJ, Brocia RW and Fisher EA. The unstirred water layer as a site of control of apolipoprotein B secretion. J Biol Chem. 1990; 265: 16741-16744

30) Twisk J, Gillian-Daniel DL, Tebon A, Wang L, Barrett PHR and Attie AD. The role of the LDL receptor in apolipoprotein B secretion. Journal of Clinical Investigation. 2000; 105: 521-532

31) Costet P, Cariou B, Lambert G, Lalanne F, Lardeux B, Jarnoux AL, Grefhorst A, Staels B and Krempf M. Hepatic PCSK9 expression is regulated by nutritional status via insulin and sterol regulatory element-binding protein 1c. J Biol Chem. 2006; 281: 6211-6218

32) Tavori H, Giunzioni I, Predazzi IM, Plubell D, Shivinsky A, Miles J, Devay RM, Liang H, Rashid S, Linton MF and Fazio S. Human PCSK9 promotes hepatic lipogenesis and atherosclerosis development via apoE- and LDLRmediated mechanisms. Cardiovasc Res. 2016; 110: 268278

33) Sniderman AD, Qi Y, Ma CI, Wang RH, Naples M, Baker C, Zhang J, Adeli K and Kiss RS. Hepatic cholesterol homeostasis: is the low-density lipoprotein pathway a regulatory or a shunt pathway? Arterioscler Thromb Vasc Biol. 2013; 33: 2481-2490

34) Le May C, Kourimate S, Langhi C, Chetiveaux M, Jarry A, Comera C, Collet X, Kuipers F, Krempf M, Cariou B and Costet P. Proprotein convertase subtilisin kexin type 9 null mice are protected from postprandial triglyceridemia. Arterioscler Thromb Vasc Biol. 2009; 29: 684-690
35) Rashid S, Tavori H, Brown PE, Linton MF, He J, Giunzioni I and Fazio S. Proprotein convertase subtilisin kexin type 9 promotes intestinal overproduction of triglyceriderich apolipoprotein B lipoproteins through both low-density lipoprotein receptor-dependent and -independent mechanisms. Circulation. 2014; 130: 431-441

36) Levy E, Ben Djoudi Ouadda A, Spahis S, Sane AT, Garofalo C, Grenier E, Emonnot L, Yara S, Couture P, Beaulieu JF, Menard D, Seidah NG and Elchebly M. PCSK9 plays a significant role in cholesterol homeostasis and lipid transport in intestinal epithelial cells. Atherosclerosis. 2013; 227: 297-306

37) Gillian-Daniel DL, Bates PW, Tebon A and Attie AD. Endoplasmic reticulum localization of the low density lipoprotein receptor mediates presecretory degradation of apolipoprotein B. Proc Natl Acad Sci U S A. 2002; 99: 4337-4342

38) Albers JJ, Kennedy H and Marcovina SM. Evidence that $\mathrm{Lp}[\mathrm{a}]$ contains one molecule of apo[a] and one molecule of apoB: evaluation of amino acid analysis data. J Lipid Res. 1996; 37: 192-196

39) Danesh J, Collins R and Peto R. Lipoprotein(a) and coronary heart disease. Meta-analysis of prospective studies. Circulation. 2000; 102: 1082-1085

40) Kamstrup PR, Tybjaerg-Hansen A, Steffensen R and Nordestgaard BG. Genetically elevated lipoprotein(a) and increased risk of myocardial infarction. JAMA. 2009; 301: 2331-2339

41) Kamstrup PR, Tybjærg-Hansen A, Steffensen R and Nordestgaard BG. GEnetically elevated lipoprotein(a) and increased risk of myocardial infarction. JAMA. 2009; 301: 2331-2339

42) Li Y, Luke MM, Shiffman D and Devlin JJ. Genetic variants in the apolipoprotein(a) gene and coronary heart disease. Circ Cardiovasc Genet. 2011; 4: 565-573

43) Lamon-Fava S, Marcovina SM, Albers JJ, Kennedy H, Deluca C, White CC, Cupples LA, McNamara JR, Seman LJ, Bongard V and Schaefer EJ. Lipoprotein(a) levels, apo(a) isoform size, and coronary heart disease risk in the Framingham Offspring Study. J Lipid Res. 2011; 52: 1181-1187

44) Raal FJ, Giugliano RP, Sabatine MS, Koren MJ, Langslet G, Bays H, Blom D, Eriksson M, Dent R, Wasserman SM, Huang F, Xue A, Albizem M, Scott R and Stein EA. Reduction in lipoprotein(a) with PCSK9 monoclonal antibody evolocumab (AMG 145): a pooled analysis of more than 1,300 patients in 4 phase II trials. J Am Coll Cardiol. 2014; 63: 1278-1288

45) Gaudet D, Kereiakes DJ, McKenney JM, Roth EM, Hanotin C, Gipe D, Du Y, Ferrand AC, Ginsberg HN and Stein EA. Effect of alirocumab, a monoclonal proprotein convertase subtilisin/kexin 9 antibody, on lipoprotein(a) concentrations (a pooled analysis of 150 $\mathrm{mg}$ every two weeks dosing from phase 2 trials). Am J Cardiol. 2014; 114: 711-715

46) Rader DJ, Mann WA, Cain W, Kraft HG, Usher D, Zech LA, Hoeg JM, Davignon J, Lupien P and Grossman M. The low density lipoprotein receptor is not required for normal catabolism of $\mathrm{Lp}(\mathrm{a})$ in humans. The Journal of Clinical Investigation. 95: 1403-1408

47) Villard EF, Thedrez A, Blankenstein J, Croyal M, Tran 
T-T-T, Poirier B, Le Bail J-C, Illiano S, Nobécourt E, Krempf M, Blom DJ, Marais AD, Janiak P, Muslin AJ, Guillot E and Lambert G. PCSK9 Modulates the Secretion But Not the Cellular Uptake of Lipoprotein(a) Ex Vivo. An Effect Blunted by Alirocumab. 2016; 1: 419427

48) Tavori H, Christian D, Minnier J, Plubell D, Shapiro MD, Yeang C, Giunzioni I, Croyal M, Duell PB, Lambert G, Tsimikas S and Fazio S. PCSK9 Association With Lipoprotein(a). Circulation Research. 2016; 119: 29-35

49) Ferri N, Tibolla G, Pirillo A, Cipollone F, Mezzetti A, Pacia S, Corsini A and Catapano AL. Proprotein convertase subtilisin kexin type 9 (PCSK9) secreted by cultured smooth muscle cells reduces macrophages LDLR levels. Atherosclerosis. 2012; 220: 381-386

50) Ding Z, Liu S, Wang X, Deng X, Fan Y, Sun C, Wang Y and Mehta JL. Hemodynamic shear stress via ROS modulates PCSK9 expression in human vascular endothelial and smooth muscle cells and along the mouse aorta. Antioxid Redox Signal. 2015; 22: 760-771

51) Ferri N, Marchiano S, Tibolla G, Baetta R, Dhyani A, Ruscica M, Uboldi P, Catapano AL and Corsini A. PCSK9 knock-out mice are protected from neointimal formation in response to perivascular carotid collar placement. Atherosclerosis. 2016; 253: 214-224

52) Mehta JL, Sanada N, Hu CP, Chen J, Dandapat A, Sugawara F, Satoh H, Inoue K, Kawase Y, Jishage K, Suzuki H, Takeya M, Schnackenberg L, Beger R, Hermonat PL, Thomas $M$ and Sawamura T. Deletion of LOX-1 reduces atherogenesis in LDLR knockout mice fed high cholesterol diet. Circ Res. 2007; 100: 1634-1642

53) Ding Z, Liu S, Wang X, Dai Y, Khaidakov M, Deng X, Fan Y, Xiang D and Mehta JL. LOX-1, mtDNA damage, and NLRP3 inflammasome activation in macrophages: implications in atherogenesis. Cardiovasc Res. 2014; 103: 619-628

54) Ding Z, Liu S, Wang X, Deng X, Fan Y, Shahanawaz J, Shmookler Reis RJ, Varughese KI, Sawamura T and Mehta JL. Cross-talk between LOX-1 and PCSK9 in vascular tissues. Cardiovasc Res. 2015; 107: 556-567

55) Herbert B, Patel D, Waddington SN, Eden ER, McAleenan A, Sun XM and Soutar AK. Increased secretion of lipoproteins in transgenic mice expressing human D374Y PCSK9 under physiological genetic control. Arterioscler Thromb Vasc Biol. 2010; 30: 1333-1339

56) Roche-Molina M, Sanz-Rosa D, Cruz FM, Garcia-Prieto J, Lopez S, Abia R, Muriana FJ, Fuster V, Ibanez B and Bernal JA. Induction of sustained hypercholesterolemia by single adeno-associated virus-mediated gene transfer of mutant hPCSK9. Arterioscler Thromb Vasc Biol. 2015; 35: 50-59

57) Denis M, Marcinkiewicz J, Zaid A, Gauthier D, Poirier S, Lazure C, Seidah NG and Prat A. Gene inactivation of proprotein convertase subtilisin/kexin type 9 reduces atherosclerosis in mice. Circulation. 2012; 125: 894-901

58) Ason B, van der Hoorn JW, Chan J, Lee E, Pieterman EJ, Nguyen KK, Di M, Shetterly S, Tang J, Yeh WC, Schwarz M, Jukema JW, Scott R, Wasserman SM, Princen HM and Jackson S. PCSK9 inhibition fails to alter hepatic LDLR, circulating cholesterol, and atherosclerosis in the absence of ApoE. J Lipid Res. 2014; 55: 2370-2379
59) Fan D, Yancey PG, Qiu S, Ding L, Weeber EJ, Linton MF and Fazio S. Self-association of human PCSK9 correlates with its LDLR-degrading activity. Biochemistry. 2008; 47: 1631-1639

60) Tavori H, Fan D, Blakemore JL, Yancey PG, Ding L, Linton MF and Fazio S. Serum proprotein convertase subtilisin/kexin type 9 and cell surface low-density lipoprotein receptor: evidence for a reciprocal regulation. Circulation. 2013; 127: 2403-2413

61) Dubuc G, Tremblay M, Pare G, Jacques H, Hamelin J, Benjannet S, Boulet L, Genest J, Bernier L, Seidah NG and Davignon J. A new method for measurement of total plasma PCSK9: clinical applications. J Lipid Res. 2010; 51: 140-149

62) Lambert G, Chatelais M, Petrides F, Passard M, Thedrez A, Rye KA, Schwahn U, Gusarova V, Blom DJ, Sasiela W and Marais AD. Normalization of low-density lipoprotein receptor expression in receptor defective homozygous familial hypercholesterolemia by inhibition of PCSK9 with alirocumab. J Am Coll Cardiol. 2014; 64: 22992300

63) Giunzioni I, Tavori H, Covarrubias R, Major AS, Ding L, Zhang Y, DeVay RM, Hong L, Fan D, Predazzi IM, Rashid S, Linton MF and Fazio S. Local effects of human PCSK9 on the atherosclerotic lesion. J Pathol. 2016; 238: $52-62$

64) Guo J, Bot I, de Nooijer R, Hoffman SJ, Stroup GB, Biessen EA, Benson GM, Groot PH, Van Eck M and Van Berkel TJ. Leucocyte cathepsin K affects atherosclerotic lesion composition and bone mineral density in low-density lipoprotein receptor deficient mice. Cardiovasc Res. 2009; 81: 278-285

65) Shah PK. Inflammation and plaque vulnerability. Cardiovasc Drugs Ther. 2009; 23: 31-40

66) Overton CD, Yancey PG, Major AS, Linton MF and Fazio S. Deletion of macrophage LDL receptor-related protein increases atherogenesis in the mouse. Circ Res. 2007; 100: 670-677

67) Chan DC, Pang J, McQuillan BM, Hung J, Beilby JP, Barrett PH and Watts GF. Plasma Proprotein Convertase Subtilisin Kexin Type 9 as a Predictor of Carotid Atherosclerosis in Asymptomatic Adults. Heart Lung Circ. 2016; 25: $520-525$

68) Lee CJ, Lee YH, Park SW, Kim KJ, Park S, Youn JC, Lee $\mathrm{SH}$, Kang SM and Jang Y. Association of serum proprotein convertase subtilisin/kexin type 9 with carotid intima media thickness in hypertensive subjects. Metabolism. 2013; 62: 845-850

69) Huijgen R, Fouchier SW, Denoun M, Hutten BA, Vissers MN, Lambert G and Kastelein JJ. Plasma levels of PCSK9 and phenotypic variability in familial hypercholesterolemia. J Lipid Res. 2012; 53: 979-983

70) Zhu YM, Anderson TJ, Sikdar K, Fung M, McQueen MJ, Lonn EM and Verma S. Association of Proprotein Convertase Subtilisin/Kexin Type 9 (PCSK9) With Cardiovascular Risk in Primary Prevention. Arterioscler Thromb Vasc Biol. 2015; 35: 2254-2259

71) Alonso R, Mata P, Muniz O, Fuentes-Jimenez F, Diaz JL, Zambon D, Tomas M, Martin C, Moyon T, Croyal M, Thedrez A and Lambert G. PCSK9 and lipoprotein (a) levels are two predictors of coronary artery calcification in 
asymptomatic patients with familial hypercholesterolemia. Atherosclerosis. 2016; 254: 249-253

72) Cheng JM, Oemrawsingh RM, Garcia-Garcia HM, Boersma E, van Geuns RJ, Serruys PW, Kardys I and Akkerhuis KM. PCSK9 in relation to coronary plaque inflammation: Results of the ATHEROREMO-IVUS study. Atherosclerosis. 2016; 248: 117-122

73) Xie W, Liu J, Wang W, Wang M, Qi Y, Zhao F, Sun J, Liu J, Li Y and Zhao D. Association between plasma PCSK9 levels and 10-year progression of carotid atherosclerosis beyond LDL-C: A cohort study. Int J Cardiol. 2016; 215: 293-298

74) Nicholls SJ, Puri R, Anderson T, Ballantyne CM, Cho L, Kastelein JJ, Koenig W, Somaratne R, Kassahun H, Yang J, Wasserman SM, Scott R, Ungi I, Podolec J, Ophuis AO, Cornel JH, Borgman M, Brennan DM and Nissen SE. Effect of Evolocumab on Progression of Coronary Disease in Statin-Treated Patients: The GLAGOV Randomized Clinical Trial. JAMA. 2016; 316: 2373-2384

75) Ridker PM, Rifai N, Bradwin G and Rose L. Plasma proprotein convertase subtilisin/kexin type 9 levels and the risk of first cardiovascular events. Eur Heart J. 2016; 37: 554-560

76) Leander K, Malarstig A, Van't Hooft FM, Hyde C, Hellenius ML, Troutt JS, Konrad RJ, Ohrvik J, Hamsten A and de Faire U. Circulating Proprotein Convertase Subtilisin/Kexin Type 9 (PCSK9) Predicts Future Risk of Cardiovascular Events Independently of Established Risk Factors. Circulation. 2016; 133: 1230-1239

77) Werner C, Hoffmann MM, Winkler K, Bohm M and Laufs U. Risk prediction with proprotein convertase subtilisin/kexin type 9 (PCSK9) in patients with stable coronary disease on statin treatment. Vascul Pharmacol. 2014; 62: 94-102

78) Li JJ, Li S, Zhang Y, Xu RX, Guo YL, Zhu CG, Wu NQ, Qing P, Gao Y, Sun J, Liu G and Dong Q. Proprotein
Convertase Subtilisin/Kexin type 9, C-Reactive Protein, Coronary Severity, and Outcomes in Patients With Stable Coronary Artery Disease: A Prospective Observational Cohort Study. Medicine (Baltimore). 2015; 94: e2426

79) Gencer B, Montecucco F, Nanchen D, Carbone F, Klingenberg R, Vuilleumier N, Aghlmandi S, Heg D, Raber L, Auer R, Juni P, Windecker S, Luscher TF, Matter CM, Rodondi N and Mach F. Prognostic value of PCSK9 levels in patients with acute coronary syndromes. Eur Heart J. 2016; 37: 546-553

80) Vlachopoulos C, Terentes-Printzios D, Georgiopoulos G, Skoumas I, Koutagiar I, Ioakeimidis N, Stefanadis C and Tousoulis D. Prediction of cardiovascular events with levels of proprotein convertase subtilisin/kexin type 9: A systematic review and meta-analysis. Atherosclerosis. 2016; 252: 50-60

81) Tavori H, Giunzioni I, Linton MF and Fazio S. Loss of plasma proprotein convertase subtilisin/kexin 9 (PCSK9) after lipoprotein apheresis. Circ Res. 2013; 113: 12901295

82) Norata GD, Tavori H, Pirillo A, Fazio S and Catapano AL. Biology of proprotein convertase subtilisin kexin 9: beyond low-density lipoprotein cholesterol lowering. Cardiovasc Res. 2016; 112: 429-442

83) Sabatine MS, Giugliano RP, Keech A, Honarpour N, Wang H, Liu T, Wasserman SM, Scott R, Sever PS and Pedersen TR. Rationale and design of the Further cardiovascular OUtcomes Research with PCSK9 Inhibition in subjects with Elevated Risk trial. Am Heart J. 2016; 173: 94-101

84) Sabatine MS, Wasserman SM and Stein EA. PCSK9 Inhibitors and Cardiovascular Events. N Engl J Med. 2015; 373: 774-775

85) Robinson JG and Kastelein JJ. PCSK9 Inhibitors and Cardiovascular Events. N Engl J Med. 2015; 373: 774 